\title{
H $\alpha$ LINE PROFILE ASYMMETRIES AND THE CHROMOSPHERIC FLARE VELOCITY FIELD
}

\author{
D. Kuridze ${ }^{1,6}$, M. Mathioudakis ${ }^{1}$, P. J. A. Simões ${ }^{2}$, L. Rouppe Van der Voort ${ }^{2}$, M. Carlsson ${ }^{3}$, S. Jafarzadeh $^{3}$, \\ J. C. Allred ${ }^{4}$, A. F. Kowalski ${ }^{4}$, M. Kennedy ${ }^{1}$, L. Fletcher ${ }^{2}$, D. Graham ${ }^{5}$, and F. P. Keenan ${ }^{1}$ \\ Astrophysics Research Centre, School of Mathematics and Physics, Queen's University Belfast BT7 1NN, UK \\ ${ }^{2}$ SUPA School of Physics and Astronomy, University of Glasgow, Glasgow G12 8QQ, UK \\ ${ }^{3}$ Institute of Theoretical Astrophysics, University of Oslo, P.O. Box 1029 Blindern, NO-0315 Oslo, Norway \\ ${ }^{4}$ Department of Astronomy, University of Maryland, College Park, MD 20742, USA \\ ${ }^{5}$ INAF-Ossevatorio Astrofisico di Arcetri, I-50125 Firenze, Italy \\ ${ }^{6}$ Abastumani Astrophysical Observatory at Ilia State University, G. Tseretili 3, 0162, Tbilisi, Georgia \\ Received 2015 September 11; accepted 2015 October 6; published 2015 November 5
}

\begin{abstract}
The asymmetries observed in the line profiles of solar flares can provide important diagnostics of the properties and dynamics of the flaring atmosphere. In this paper the evolution of the $\mathrm{H} \alpha$ and $\mathrm{Ca}$ II $\lambda 8542$ lines are studied using high spatial, temporal, and spectral resolution ground-based observations of an M1.1 flare obtained with the Swedish $1 \mathrm{~m}$ Solar Telescope. The temporal evolution of the $\mathrm{H} \alpha$ line profiles from the flare kernel shows excess emission in the red wing (red asymmetry) before flare maximum and excess in the blue wing (blue asymmetry) after maximum. However, the Ca II $\lambda 8542$ line does not follow the same pattern, showing only a weak red asymmetry during the flare. RADYN simulations are used to synthesize spectral line profiles for the flaring atmosphere, and good agreement is found with the observations. We show that the red asymmetry observed in $\mathrm{H} \alpha$ is not necessarily associated with plasma downflows, and the blue asymmetry may not be related to plasma upflows. Indeed, we conclude that the steep velocity gradients in the flaring chromosphere modify the wavelength of the central reversal in the $\mathrm{H} \alpha$ line profile. The shift in the wavelength of maximum opacity to shorter and longer wavelengths generates the red and blue asymmetries, respectively.
\end{abstract}

Key words: radiative transfer - Sun: chromosphere - Sun: flares

\section{INTRODUCTION}

It is widely accepted that the vast majority of the solar flare radiative energy originates in the photosphere and chromosphere. The chromospheric radiation is dominated by hydrogen, calcium, and magnesium lines, plus hydrogen continua, providing vital diagnostics on energy deposition rates in the lower atmosphere. One of the main characteristics of the flaring chromosphere is the centrally reversed $\mathrm{H} \alpha$ emission line profile with asymmetric red and blue wings (see the review by Berlicki 2007). Earlier observations have shown that the red asymmetry in the $\mathrm{H} \alpha$ line profile is more typical and is observed frequently during the impulsive phase of the flare (Švestka 1976; Tang 1983; Ichimoto \& Kurokawa 1984; Wülser \& Marti 1989; Falchi et al. 1997). High-resolution spectroscopy indicates that the red asymmetry dominates over the ribbons during the flare (Asai et al. 2012; Deng et al. 2013; Huang et al. 2014). However, blue asymmetries have also been reported, and these occur mainly in the early stages of the flare (Švestka et al. 1962; Severny 1968; Heinzel et al. 1994; Mein et al. 1997; Yu \& Gan 2006). Furthermore, blue and red asymmetries at different positions in the same flare ribbon and the reversal of asymmetry at the same position in the ribbon have also been reported (Canfield et al. 1990a; Ji et al. 1994; Mein et al. 1997). The Ca II $\lambda 8542$ line profile, which goes into full emission during the flare, is also asymmetric ( $\mathrm{Li}$ et al. 2007), but its asymmetric properties do not necessarily coincide with those detected simultaneously in $\mathrm{H} \alpha$ (Mein et al. 1997).

Despite a number of attempts to explain the observed asymmetries, their exact nature remains unclear. Theoretical line profiles based on static models of flare chromospheres do not show asymmetric signatures (Canfield et al. 1984; Fang et al. 1993; Cheng et al. 2006). This suggests that the asymmetries are related to the velocity field in the flaring atmosphere.

The red asymmetries observed in chromospheric lines during flares are usually attributed to the downflow of cool plasma in the flaring atmosphere, a process also known as chromospheric condensation (Ichimoto \& Kurokawa 1984; Wülser \& Marti 1989; de la Beaujardiere et al. 1992). As the flare energy is deposited in the chromosphere, a strong evaporation of the heated plasma occurs (e.g., Neupert 1968; Fisher et al. 1985; Graham \& Cauzzi 2015). Conservation of momentum requires the formation of a downflow in the form of chromospheric condensation (Ichimoto \& Kurokawa 1984; Canfield et al. 1990b; Milligan \& Dennis 2009; Hudson 2011; Kerr et al. 2015). If the downflow occurs in the upper chromosphere, its effect would be a red wing absorption, which can lead to a blue asymmetry in the line profile (Gan et al. 1993; Heinzel et al. 1994). Ding \& Fang (1996) have shown that both red and blue asymmetries in the $\mathrm{H} \alpha$ line can be caused by downwardmoving plasma at different heights in the solar chromosphere. This blue asymmetry has also been interpreted in terms of chromospheric evaporation and filament activation (Canfield et al. 1990a; Huang et al. 2014).

Abbett \& Hawley (1999) computed time-dependent $\mathrm{H} \alpha$ and $\mathrm{Ca}$ II $\mathrm{K}$ line profiles with the radiative hydrodynamic code (RADYN; Carlsson \& Stein 1997) for weak (F10) and strong (F11) flare runs and showed that asymmetries could be produced by the strong velocity gradients generated during the flare. The velocity gradients create differences in the opacity between the red and blue wings of $\mathrm{H} \alpha$, and the sign of the gradient determines whether the asymmetric emission appears to the blue or red side of the line profile. This process was first reported in the pioneering work of Carlsson \& Stein 
(1997), who studied the effects of acoustic shocks on the formation of the $\mathrm{Ca}$ II $\mathrm{H}$ line in a nonflaring atmosphere. More recently, Rubio da Costa et al. (2014) made a direct comparison between observations and RADYN simulations of an M3.0 flare. Their observed and synthesized $\mathrm{H} \alpha$ and $\mathrm{Ca}$ II $\lambda 8542$ line profiles are asymmetric, especially during the early stages of the flare.

In this paper we present high temporal, spatial, and spectral resolution imaging spectroscopy of an M1.1 solar flare. We study the evolution of the $\mathrm{H} \alpha$ and $\mathrm{Ca}$ II $\lambda 8542$ line profiles of the flare kernel and compare these with synthesized line profiles obtained with a radiative hydrodynamic simulation. The line contribution functions and the velocity field in the simulated atmosphere allow us to disentangle the nature of the observed line asymmetries.

\section{OBSERVATIONS AND DATA REDUCTION}

The observations presented in this paper were undertaken between 16:27 and 17:27 UT on 2014 September 6 with the CRisp Imaging SpectroPolarimeter (CRISP; Scharmer 2006; Scharmer et al. 2008) instrument, mounted on the Swedish $1 \mathrm{~m}$ Solar Telescope (SST; Scharmer et al. 2003a) on La Palma. Adaptive optics were used throughout the observations, consisting of a tip-tilt mirror and an 85-electrode deformable mirror setup that is an upgrade of the system described in Scharmer et al. (2003b). The observations were composed of spectral imaging in the $\mathrm{H} \alpha \lambda 6563$ and $\mathrm{Ca}$ II $\lambda 8542$ lines and Stokes $V$ maps in $\mathrm{Fe}_{\mathrm{I}} \lambda 6302-0.048$. All data were reconstructed with the Multi-object Multi-frame Blind Deconvolution technique (MOMFBD; van Noort et al. 2005). We applied the CRISP data reduction pipeline as described in de la Cruz Rodríguez et al. (2015). Our spatial sampling is 0 ". 057 pixel $^{-1}$, and the spatial resolution is close to the diffraction limit of the telescope for many images in the time series. The $\mathrm{H} \alpha$ line scan consists of 15 positions symmetrically sampled with $0.2 \AA$ steps from line core, with the $\mathrm{Ca}$ II $\lambda 8542$ scan consisting of 25 line positions with $0.1 \AA$ steps. A full spectral scan had an acquisition time of $11.54 \mathrm{~s}$, which is the temporal cadence of the time series. We made use of CRISPEX (Vissers \& Rouppe van der Voort 2012), a versatile widget-based tool for effective viewing and exploration of multidimensional imaging spectroscopy data.

The flare was also observed by RHESSI (Lin et al. 2002) and the Gamma-ray Burst Monitor (GBM; Meegan et al. 2009) on Fermi. In Section 4 we describe the analysis of RHESSI data, noting that similar results were found from Fermi/GBM. The hard X-ray (HXR) spectral analysis was performed using OSPEX (Schwartz et al. 2002) to estimate the power $P_{\text {nth }}$ deposited in the chromosphere by the nonthermal electrons, assuming a thick-target model.

\section{3. $\mathrm{H} \alpha$ AND CA II $\lambda 8542$ LINE ASYMMETRIES}

The two-ribbon M1.1 flare was observed in NOAA Active Region 12157 located at heliocentric coordinates $\sim\left(-732^{\prime \prime}\right.$, $\left.-302^{\prime \prime}\right)$. Figure 1 shows the flare images in $\mathrm{H} \alpha$ and $\mathrm{Ca}$ II $\lambda 8542$ line core and wing positions, together with a photospheric $\mathrm{Fe}_{\mathrm{I}} \lambda 6302-0.048$ running difference image. An examination of the temporal evolution of the flare ribbons suggests that they remain stationary in the image plane during the flare. We identified a bright kernel in the CRISP images, highlighted with the red boxes in Figure 1, which is co-spatial with the footpoint of the coronal loops observed in AIA $94 \AA$ images (Figure 1). Light curves of the region show that the emission from the $\mathrm{H} \alpha$ and $\mathrm{Ca}$ II $\lambda 8542$ line wings and the photospheric $\mathrm{Fe}_{\mathrm{I}}$ line between 16:54 and 16:56 UT increases, peaking at 16:55:24 (Figure 2). We find no evidence for time delays between the different wavelengths in excess of the $11.54 \mathrm{~s}$ temporal resolution element.

In Figure 3 we show the $\mathrm{H} \alpha$ and $\mathrm{Ca}$ II $\lambda 8542$ line profiles averaged over the $\sim 2^{\prime \prime} \times 2^{\prime \prime}$ area marked with the red boxes in Figure 1. Shortly after the flare onset, the flaring chromosphere produces a wide $\mathrm{H} \alpha$ emission profile with a central reversal. The temporal evolution of the centrally reversed $\mathrm{H} \alpha$ profiles shows excess emission in the red wing (red asymmetry) with nearly unshifted line center before the flare maximum (top left panel of Figure 3). However, after the flare maximum the blue peak becomes higher than the red peak, and hence a blue asymmetry is formed (top right panel of Figure 3). Furthermore, the line centers of these blue asymmetric $\mathrm{H} \alpha$ profiles are shifted to longer wavelengths (top right panel of Figures 3 and 4).

To quantify the asymmetry of the $\mathrm{H} \alpha$ line profiles, we use a technique similar to that described in Mein et al. (1997) and De Pontieu et al. (2009). We identify the central wavelength of the red and blue peaks and compute the intensities in these positions integrated over a $\pm 0.2 \AA$ interval (left panel of Figure 4). These intensities are given as

$$
I_{B}=\sum_{\lambda_{0 B}-\delta \lambda}^{\lambda_{0 B}+\delta \lambda} I_{\lambda}, \quad I_{R}=\sum_{\lambda_{0 R}-\delta \lambda}^{\lambda_{0 R}+\delta \lambda} I_{\lambda},
$$

where $\lambda_{O B}$ and $\lambda_{O R}$ are the centroids of the blue and red peaks, respectively, and $\delta \lambda$ the wavelength range over which the intensities are integrated. The ratio $I_{B} / I_{R}$ allows us to study the evolution of the asymmetries as a function of time (right panel of Figure 4) and is normalized to the mean intensity ratios of the nonflaring atmospheric profile computed over the same wavelength range, such that $I_{B} / I_{R} \approx 1$ corresponds to no asymmetries in the $\mathrm{H} \alpha$ line profile. Figure 4 (right panel) shows that at $\sim 16: 50$ UT a strong red asymmetry begins to appear, increasing to the flare maximum $(\sim 16: 55 \mathrm{UT})$. The red asymmetry then decreases very quickly (within about 2 minutes), with the blue asymmetry appearing and maintained until the end of the observations.

The $\mathrm{Ca}$ II $\lambda 8542$ line has a slightly redshifted emission profile of about $0.1 \AA$ (corresponding to a velocity of $\sim 3.5 \mathrm{~km} \mathrm{~s}^{-1}$ ), and in contrast to $\mathrm{H} \alpha$, it does not reveal the asymmetry reversal during the flare (bottom panels of Figure 3).

\section{HARD X-RAYS}

HXR emission is a typical signature for the presence of accelerated electrons in flares. Assuming that the electron distribution $F(E)$ has a power-law form $A E^{-\delta}$ (electrons $\mathrm{s}^{-1} \mathrm{keV}^{-1}$ ), where $A$ is the normalization factor (proportional to the total electron rate), $E_{C}$ is the low-energy cutoff, and $\delta$ is the spectral index, a lower limit for the total power contained in the distribution can be estimated from

$$
P_{\text {nth }}\left(E \geqslant E_{C}\right)=\int_{E_{C}}^{\infty} E F(E) d E \operatorname{erg~s}^{-1} \text {. }
$$

We fitted the HXR spectrum obtained by RHESSI with an isothermal plus thick-target model (as described above, using 

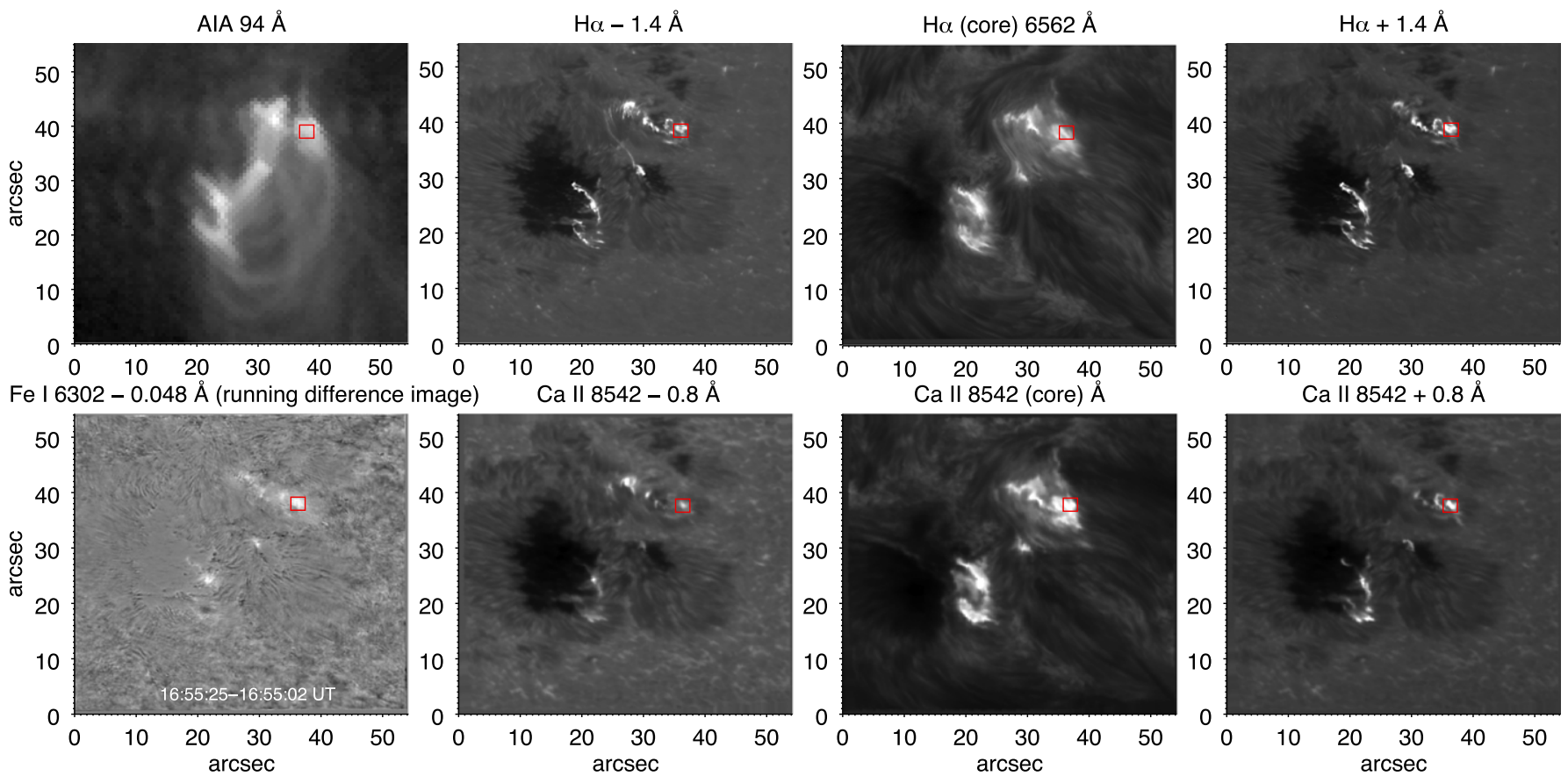

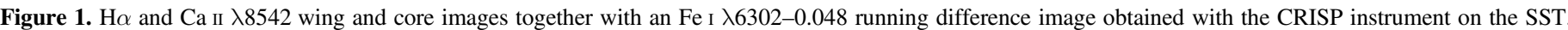

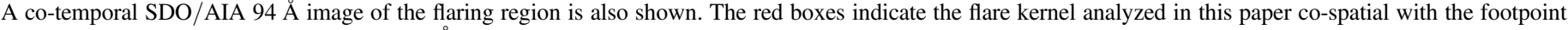
of the coronal loops observed in the AIA $94 \AA$ image.

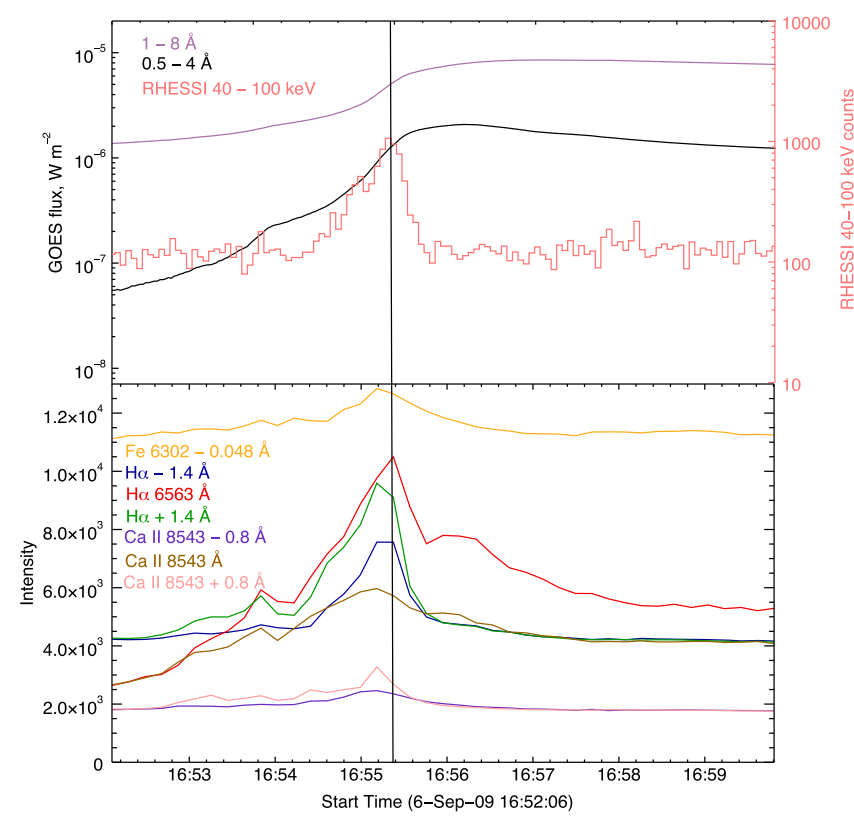

Figure 2. Top: GOES X-ray and RHESSI 40-100 keV HXR light curves. Bottom: light curves of the flaring region marked with the red boxes in Figure 1.

the OSPEX function thick2_vnorm) and calculated $P_{\text {nth }}$ throughout the impulsive phase, integrating the counts in bins of $12 \mathrm{~s}$. RHESSI front detectors $1,3,6,8$, and 9 were employed, and the fitting results for $A, \delta, E_{C}$, and $P_{\mathrm{nth}}$ are shown in Figure 5. We found $P_{\text {nth }} \approx 1.3 \times 10^{28} \mathrm{erg} \mathrm{s}^{-1}$ at the time of maximum of HXR emission, between 16:55:18 and 16:55:30 UT. To obtain the energy flux $\left(\mathrm{erg} \mathrm{s}^{-1} \mathrm{~cm}^{-2}\right)$ deposited into the chromospheric source, one divides the power of the nonthermal electrons $P_{\text {nth }}$ by the footpoint area.
The reconstructed images for the HXR emission (using CLEAN; Hurford et al. 2002; detectors 3-8, beam width factor of 1.5) show three main HXR sources in good association with the main locations of the optical and UV emission, as can be seen in Figure 6. However, the HXR sources are unresolved, and the area obtained from the RHESSI images would be an overestimate of the actual sizes of those regions. The area of the ribbons was hence determined by contouring a region with intensity above $30 \%$ of the maximum of the $\mathrm{H} \alpha-1.4 \AA$ image near the HXR peak (Figure 6). We obtained an area of $0.7 \times 10^{17} \mathrm{~cm}^{2}$. Thus, at the time of the maximum HXR emission, which also coincides with the maximum of the emission in $\mathrm{H} \alpha, \mathrm{Ca}$ II, and $\mathrm{Fe}_{\mathrm{I}}$ (see Figure 2), the average energy flux delivered by the electrons into the chromosphere is around $1.9 \times 10^{11} \mathrm{erg} \mathrm{s}^{-1} \mathrm{~cm}^{-2}$.

\section{SIMULATED LINE PROFILES}

The short duration of photospheric enhancements (Figure 2) and the stationary nature of the flare ribbon make this event a good candidate for a comparison with a dynamic model.

We use the radiative hydrodynamic code RADYN to compute $\mathrm{H} \alpha$ and $\mathrm{Ca}$ II $\lambda 8542$ line profiles (Carlsson \& Stein 1997; Allred et al. 2005). The simulation was performed for a strong beam with $F=10^{11} \mathrm{erg} \mathrm{cm}^{-2} \mathrm{~s}^{-1}$ (also known as the F11 flare) and an isotropic pitch angle distribution in the forward hemisphere with the Fokker-Planck solution to the nonthermal electron distribution (Allred et al. 2015). A constant heating flux was applied for $20 \mathrm{~s}$, and the atmosphere was allowed to relax for an additional $40 \mathrm{~s}$. We used a powerlaw index and a low-energy cutoff of $\delta=4.2$ and $E_{c}=25 \mathrm{keV}$, respectively. The beam parameters used in the model are very close to the ones estimated from the HXR data (Section 4).

Figure 7 shows the temporal evolution of the synthesized $\mathrm{H} \alpha$ profiles from the F11 flare model, which indicates a behavior similar to that in the observations. Increased emission in the red 

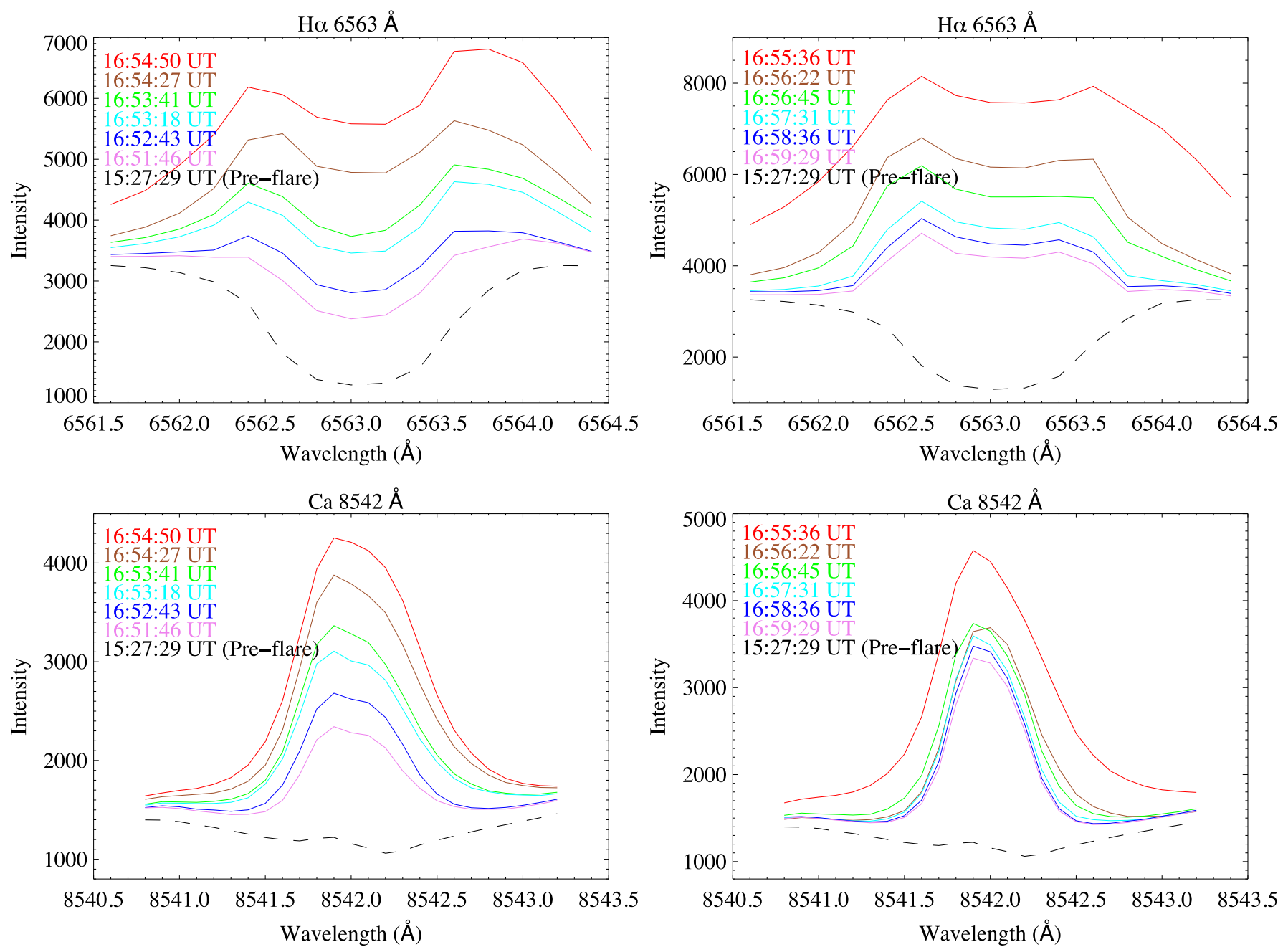

Figure 3. Temporal evolution of the $\mathrm{H} \alpha$ and $\mathrm{Ca}$ II $\lambda 8542$ line profiles before the flare maximum (left panels) and after the flare maximum (right panels) for the region outlined with the red boxes in Figure 1.
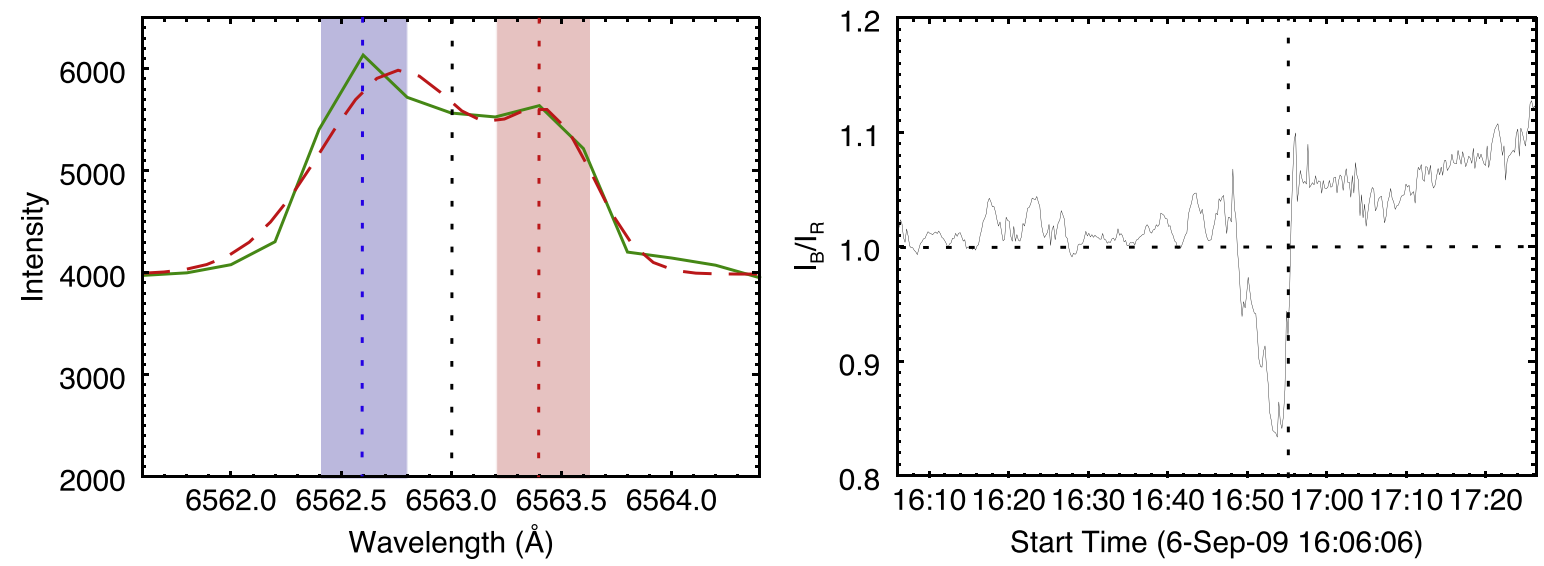

Figure 4. Left: same $\mathrm{H} \alpha$ line profile presented in the top left panel of Figure 3 with a green line. The dashed red line shows the double-Gaussian fit. Right: evolution of line profile asymmetry, $I_{B} / I_{R}$, calculated by dividing the intensities of the blue and red peak centroid positions integrated over the $\pm 0.2 \AA$ wavelength range (red and blue areas shown in the left panel). Values of $I_{B} / I_{R}$ are normalized by the mean intensity ratios of the nonflaring atmospheric profile, such that $I_{B} / I_{R} \approx 1$ corresponds to no asymmetries in the $\mathrm{H} \alpha$ spectra.

side of the profile (i.e., red asymmetry) occurs when the $\mathrm{H} \alpha$ line center is shifted to the blue, whereas during the blue asymmetry the line center is shifted to the red. The right panel of Figure 7 shows the evolution of the line profile asymmetry, $I_{B} / I_{R}$, calculated using the method described in Section 3.
Simulated Ca II $\lambda 8542$ line profiles are in emission during the flare with an extended blue wing and a slightly blueshifted center.

To understand the formation of the asymmetric line profiles, we need to examine the line contribution functions (Carlsson \& 

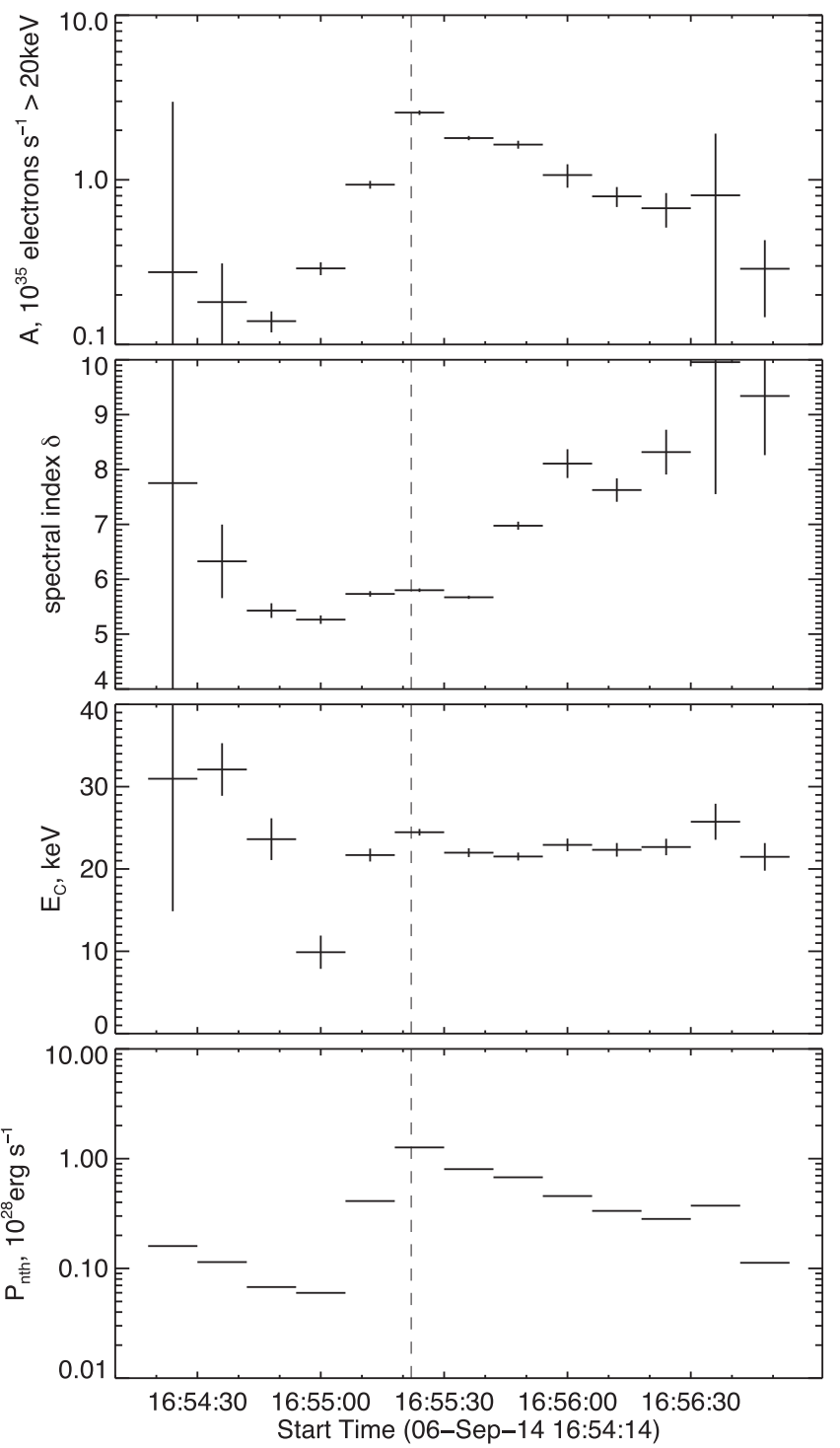

Figure 5. Fitting results of RHESSI HXR data: normalization factor $A$, spectral index $\delta$, low-energy cutoff $E_{C}$, and the total power contained in the electron distribution $P_{\mathrm{nth}}$, given by Equation (2). The vertical dashed line indicates the time of the maximum HXR emission.

Stein 1997). These are the intensities emitted in specific wavelengths as a function of height. Carlsson \& Stein (1997) introduced a formal solution of the radiative transfer equation for the emergent intensity in terms of the contribution function, $C_{I}$, as

$$
I_{\nu}=\int_{z_{0}}^{z} C_{I} d z=\int_{z_{0}}^{z} S_{\nu} \tau_{\nu} e^{-\tau_{\nu}} \chi_{\nu} / \tau_{\nu} d z
$$

where $z$ is the atmospheric height and $S_{\nu}, \tau_{\nu}$, and $\chi_{\nu}$ are the source function, optical depth, and opacity (linear extinction coefficient), respectively. Traditionally, this function is attuned to the three frequency $(\nu)$ dependent physically meaningful terms: $S_{\nu}, \tau_{\nu} e^{-\tau_{\nu}}$, and $\chi_{\nu} / \tau_{\nu}$, where $S_{\nu}$ is defined as the ratio of the emissivity over the opacity of the atmosphere. The $\tau_{\nu} e^{-\tau_{\nu}}$ term expresses an attenuation caused by optical depth and peaks near $\tau=1$, while the ratio $\chi_{\nu} / \tau_{\nu}$ is large where there are many emitting particles at low optical depths.

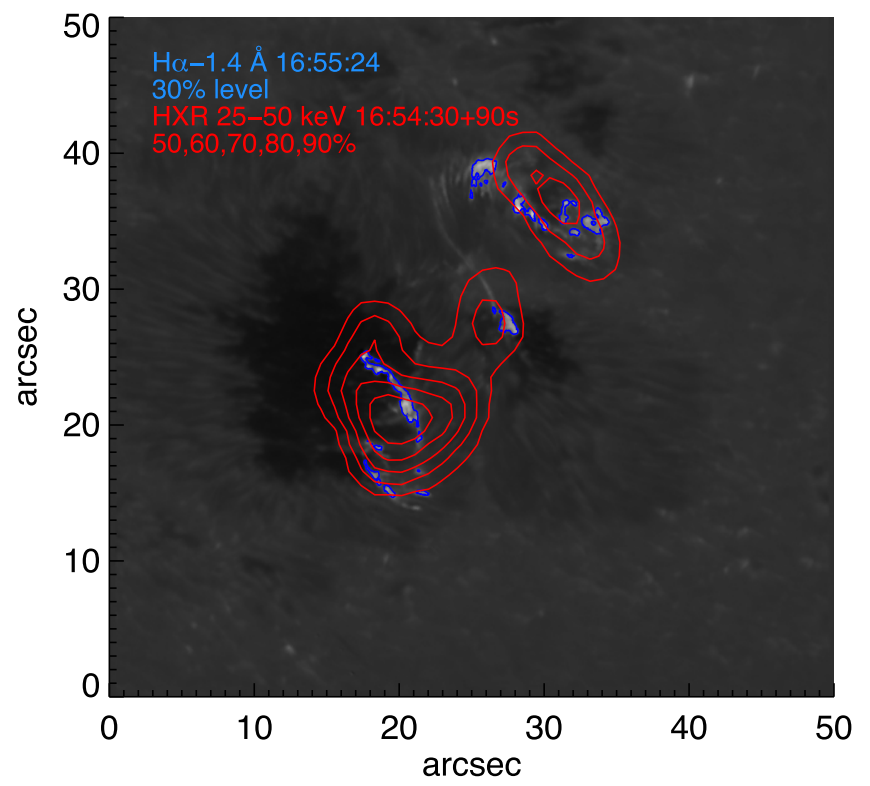

Figure 6. $\mathrm{H} \alpha-1.4 \AA$ image at the flare peak $(16: 55: 24 \mathrm{UT})$, with blue contours showing the $30 \%$ level of the image maximum, overlaid with a RHESSI 25-50 keV reconstructed image, shown as red contours at 50\%, 60\%, $70 \%, 80 \%$, and $90 \%$ of the maximum.

In Figures $8-10$ we present line contribution functions for $\mathrm{H} \alpha$ and $\mathrm{Ca}$ II $\lambda 8542$ using the classic diagrams introduced by Carlsson \& Stein (1997). The diagrams are plotted in inverse grayscale, with darker shades showing higher intensities. Line profiles are shown as dark lines in the bottom right panels. Red lines in the diagrams show the height at $\tau=1$, while the vertical velocity structure of the plasma is plotted as a blue dashed line. We note that positive values for velocities correspond to plasma upflows. The source functions (green lines) and Planck functions (brown lines) at line center are overplotted in the bottom left panels to show the height of nonLTE decoupling in the atmosphere.

Figure 8 contains a snapshot of the parameters that contribute to the $\mathrm{H} \alpha$ line profile and the formation of the red asymmetry $3 \mathrm{~s}$ into the F11 flare run. It shows that the line wings and line core are formed at $0.9-1.0 \mathrm{Mm}$ and 1.1-1.18 Mm, respectively. The line source function has a local maximum near the wing formation height and decreases toward the upper layers where the line core is formed. This leads to increased emission in the line wings, relative to the line core, hence the centrally reversed line profiles. The velocity structure of the flaring atmosphere shows that the region of $\mathrm{H} \alpha$ wing formation has a weak downflow velocity of around $1-3 \mathrm{~km} \mathrm{~s}^{-1}$. However, the region of core formation has a moderately strong upflow, with a peak value of $\sim 15 \mathrm{~km} \mathrm{~s}^{-1}$ at 1.17 Mm (Figure 8). This upflow shifts the line core of the centrally reversed $\mathrm{H} \alpha$ profile to the blue (bottom right panel). Therefore, the central wavelength of maximum opacity is also shifted to the blue. Consequently, the $\tau=1$ layer is formed higher and the $\chi_{\nu} / \tau_{\nu}$ is smaller in the blue wing, and hence blue wing photons are absorbed more easily compared to their red wing counterparts.

Figure 9 shows similar diagrams $14 \mathrm{~s}$ into the F11 flare run. Now the $\mathrm{H} \alpha$ line core is formed deeper into the atmosphere, indicating that the chromosphere is compressed by the transition region, which moves downward from 1.18 to $1.08 \mathrm{Mm}$ over about $10 \mathrm{~s}$ (see Figures 8 and 9). At the core 

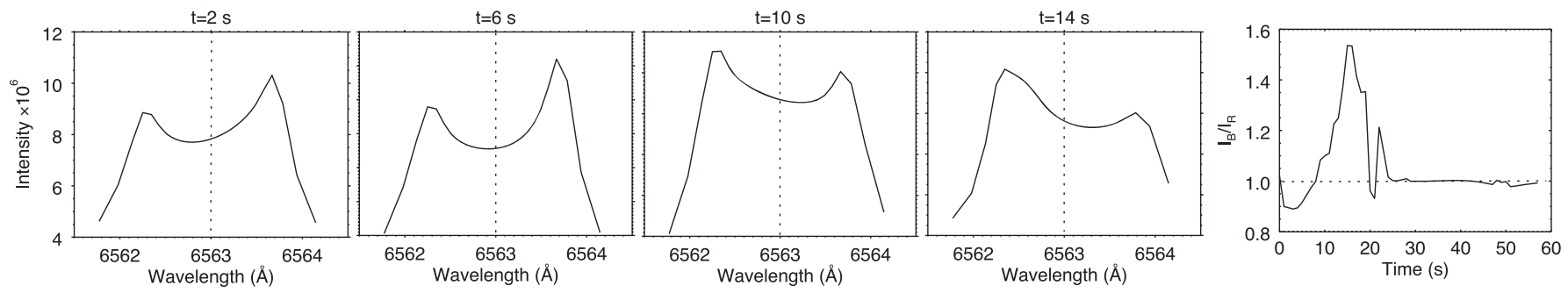

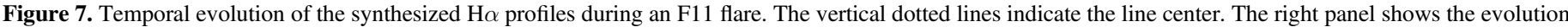
of line profile asymmetry, $I_{B} / I_{R}$, calculated with the method described in Section 3 .

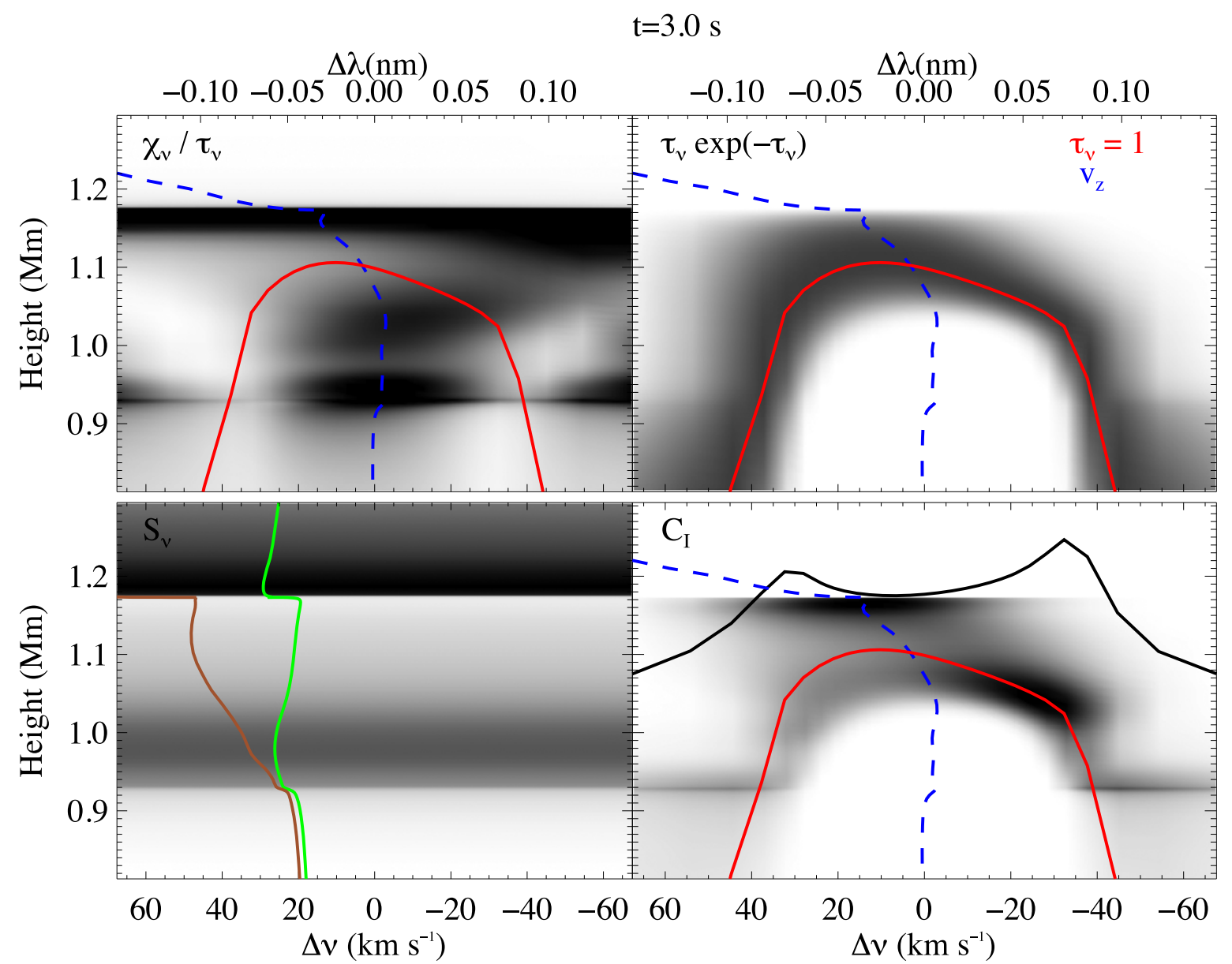

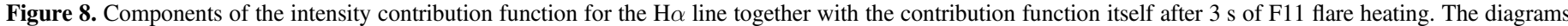

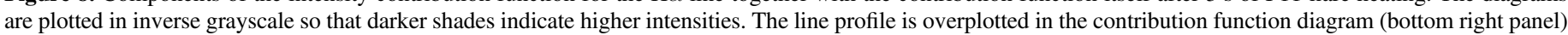

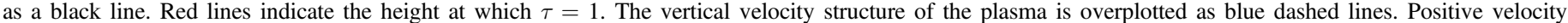
corresponds to plasma upflows. The source function (green line) and Planck function (brown line) at the line center are overplotted in the bottom left panels.

formation height (at around $1.08 \mathrm{Mm}$ ) the atmosphere has developed a moderately strong downflow (condensation) of about $-18 \mathrm{~km} \mathrm{~s}^{-1}$ (downward velocities are negative) with a negative velocity gradient. This shifts the central reversal (maximum opacity) to the red side (Figure 9). The higher-lying plasma of the line core absorbs red photons, increasing the opacity in the red wing. Therefore, the red wing photons produced between 0.9 and $1.05 \mathrm{Mm}$ can no longer escape as readily as at $t=3 \mathrm{~s}$ because the $\tau=1$ curve has shifted to the red owing to the downflowing material above. As a result, the $\tau=1$ curve is formed higher in the atmosphere at the red wing and $\chi_{\nu} / \tau_{\nu}$ is larger at the blue side of the core. This makes the contribution function, and hence the emission, larger in the blue wing, and the blue asymmetry is established (bottom right panel of Figure 9).

Figure 10 shows a similar diagram for the $\mathrm{Ca}$ II $\lambda 8542$ line $14 \mathrm{~s}$ into the F11 flare. This line is much narrower and formed deeper into the atmosphere (below $1 \mathrm{Mm}$ ) than $\mathrm{H} \alpha$. The velocity field along this height is very weak $\left(\sim \pm 1-2 \mathrm{~km} \mathrm{~s}^{-1}\right)$ during the flare, and the line source function has a maximum at the height of core formation $(\sim 0.9 \mathrm{Mm})$ (bottom left panel), so the higher emission originates from the line core. An upflow velocity field below $\sim 0.8 \mathrm{Mm}$ produces a slightly extended blue wing and creates a blue asymmetry. However, a weak downflow at the height of core formation $(\sim 0.9 \mathrm{Mm})$ shifts the line center to the red. 


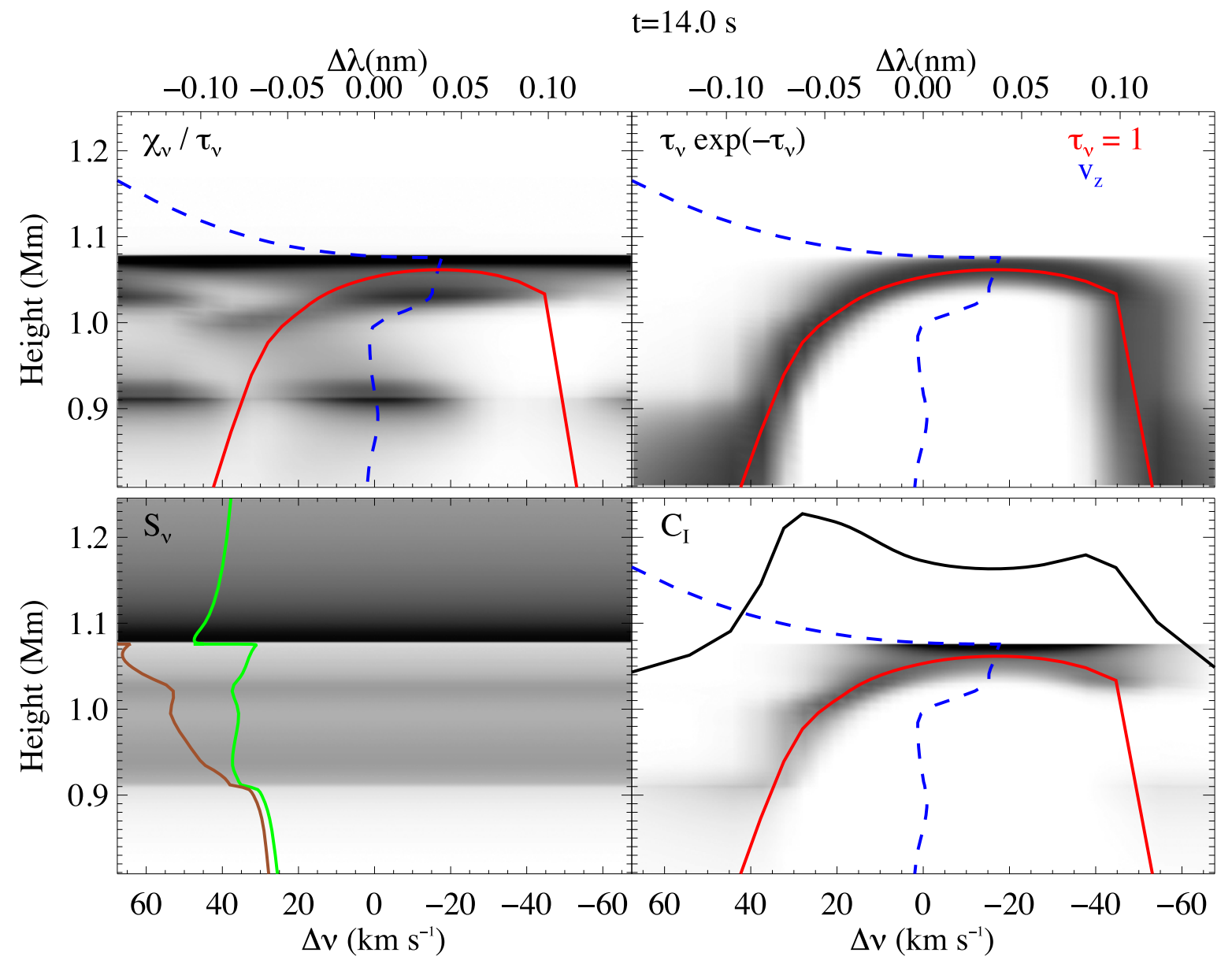

Figure 9. Components of the intensity contribution function together with the contribution function itself for the $\mathrm{H} \alpha$ line after $14 \mathrm{~s}$ of F11 flare heating. The line styles and notations are the same as in Figure 6.

\section{DISCUSSION AND CONCLUSION}

The temporal evolution of the $\mathrm{H} \alpha$ line profile of the flare kernel revealed excess emission in the red wing with an unshifted line core before flare maximum and excess emission in the blue wing with a redshifted line core after flare maximum. Numerical simulations show that in an atmosphere without velocity fields, the centrally reversed $\mathrm{H} \alpha$ profile is symmetric with respect to the line core (Canfield et al. 1984; Fang et al. 1993; Cheng et al. 2006). However, the dynamic models take into account the mass motions associated with the processes of evaporation and condensation of the flaring material, which generate strong asymmetric signatures.

We emphasize that the analysis presented here has shown that the red asymmetry observed in the $\mathrm{H} \alpha$ line profile is not necessarily associated with plasma downflows, and the blue asymmetry may not be related to plasma upflows. Thus, using these line asymmetries as a direct measure of Doppler velocities can lead to inaccurate results.

Motivated by the close match between simulations and observations, we analyzed components of intensity contribution functions for the $\mathrm{H} \alpha$ and $\mathrm{Ca}$ II $\lambda 8542$ lines (based on the approach presented in Carlsson \& Stein 1997; Abbett \& Hawley 1999). We find that in the early stages of the flare the line core of the centrally reversed $\mathrm{H} \alpha$ profile is blueshifted owing to the strong upflows at the height of core formation (bottom right panel of Figure 8). The velocity decreases downward toward the wing formation regions, producing a steep positive velocity gradient. This gradient modifies the optical depth of the atmosphere in such a way that higher-lying (core) atoms absorb photons with shorter wavelengths (blue wing photons) and the red asymmetry is formed (Figure 8). In the later stages the velocity field becomes dominated by the downflows owing to the plasma condensation with strong negative velocity gradients (Figure 9). This shifts the absorption peak (wavelength of maximum opacity) to the red (bottom right panel of Figure 9), so the higher-lying core plasma now absorbs red photons and produces a blue asymmetry.

The Ca II $\lambda 8542$ line in the F11 model is in emission and formed deeper into the atmosphere than $\mathrm{H} \alpha$. The weak velocity field along its formation height produces an extended blue wing in the line profile (bottom right panel of Figure 10), in agreement with the observations. The simulated profile shows a weak downflow at the height of core formation that shifts the line center to the red (bottom right panel of Figure 10). This is not detected in the observed spectra.

The main difference between the simulations and the observations presented in this work is that the core of the observed $\mathrm{H} \alpha$ profiles remains unshifted when the red asymmetry is detected (top left panel of Figure 3). This may be due to the presence of a much more complex velocity field with several unresolved condensation/evaporation patterns, which can produce an effectively unshifted or unresolved line core. The simulations show that when the red asymmetry is formed, there are weak downflows in the region between the 


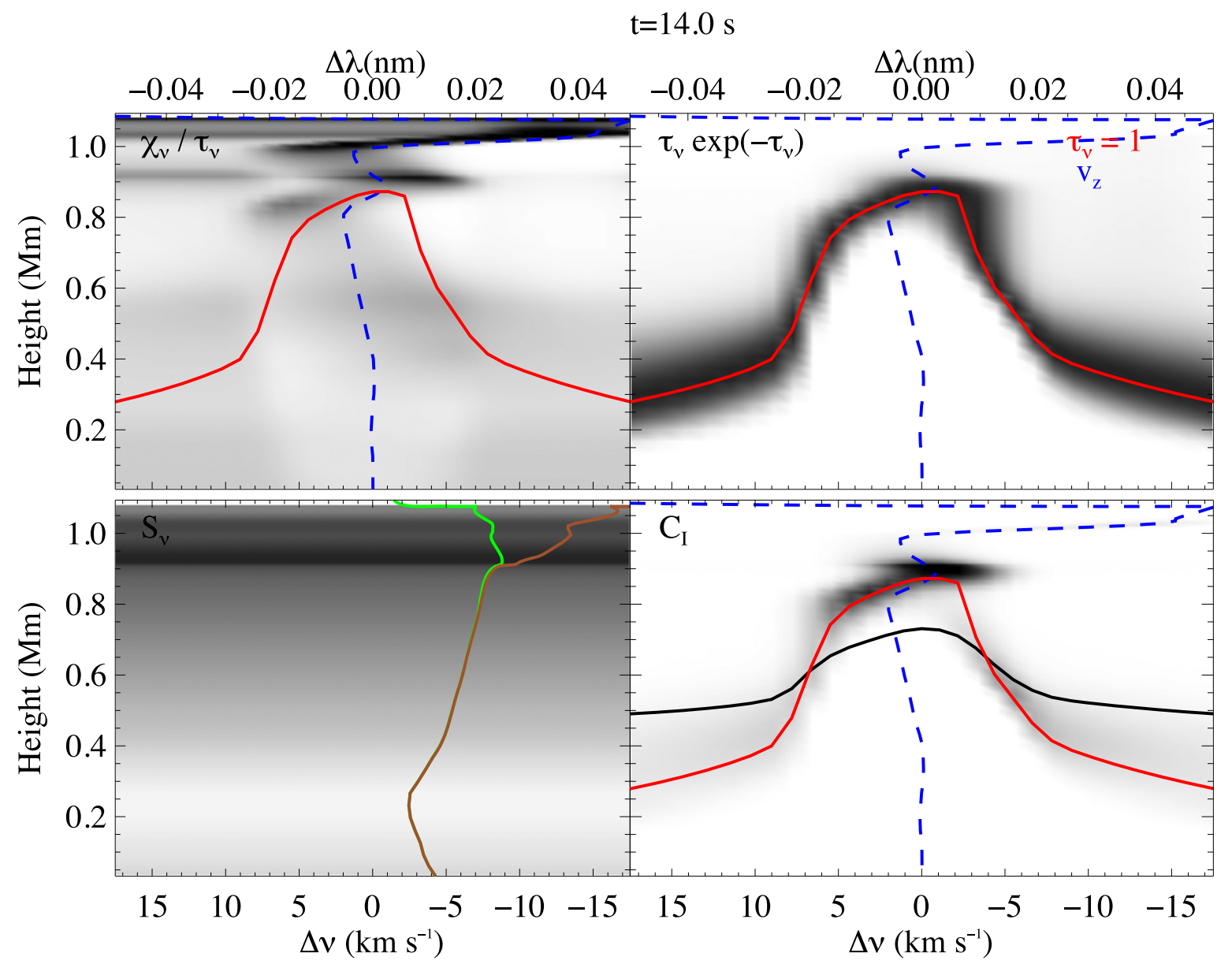

Figure 10. Components of the intensity contribution function for the $\mathrm{Ca}$ II $\lambda 8542$ line together with the contribution function itself after $14 \mathrm{~s}$ of $\mathrm{F} 11 \mathrm{flare}$ heating. The line styles and notations are the same as in Figure 6.

core and wing formation heights (see velocity field in Figure 8). However, these downflows are not strong enough to have a significant effect on the line profiles.

It should be noted that the red asymmetry in $\mathrm{H} \alpha$ begins at 16:50 (right panel of Figure 4), whereas RHESSI HXR emission starts to increase after 16:54 UT (top panel of Figure 2). This suggests that the $\mathrm{H} \alpha$ is responding to preimpulsive phase heating. In the pre-impulsive phase flare reconnection can produce direct heating instead of nonthermal particle heating. That could drive gentle, rather then explosive, chromospheric evaporation, which can produce an enhanced red wing emission in a manner similar to the one presented in Figure 8. However, the difference between simulations and observations could be related to the fact that the red asymmetry in simulations is a result of explosive evaporation produced by nonthermal particle heating, whereas in the observations the red asymmetry is suggested to be produced with a more gentle evaporation at the pre-impulsive phase heating.

Furthermore, the evolution timescale of the observed $\mathrm{H} \alpha$ line profile is longer ( $\sim 5$ minutes) than that of the simulated line profile ( $20 \mathrm{~s}$ ). Additional direct comparisons of the improved spectral resolution observations and more realistic simulations are necessary to understand the formation of spectral line asymmetries in the flaring solar chromosphere.

The research leading to these results has received funding from the European Community's Seventh Framework
Programme (FP7/2007-2013) under grant agreement No. 606862 (F-CHROMA). The Swedish $1 \mathrm{~m}$ Solar Telescope is operated on the island of La Palma by the Institute for Solar Physics (ISP) of Stockholm University in the Spanish Observatorio del Roque de los Muchachos of the Instituto de Astrofísica de Canarias.

\section{REFERENCES}

Abbett, W. P., \& Hawley, S. L. 1999, ApJ, 521, 906

Allred, J. C., Hawley, S. L., Abbett, W. P., \& Carlsson, M. 2005, ApJ, 630, 573 Allred, J. C., Kowalski, A. F., \& Carlsson, M. 2015, ApJ, 809, 104

Asai, A., Ichimoto, K., Kita, R., Kurokawa, H., \& Shibata, K. 2012, PASJ, 64, 20

Berlicki, A. 2007, in ASP Conf. Ser. 368, The Physics of Chromospheric Plasmas, ed. P. Heinzel, I. Dorotovič , \& R. J. Rutten (San Francisco, CA: ASP), 387

Canfield, R. C., Gunkler, T. A., \& Ricchiazzi, P. J. 1984, ApJ, 282, 296

Canfield, R. C., Penn, M. J., Wulser, J.-P., \& Kiplinger, A. L. 1990a, ApJ, 363,318

Canfield, R. C., Zarro, D. M., Metcalf, T. R., \& Lemen, J. R. 1990b, ApJ, 348,333

Carlsson, M., \& Stein, R. F. 1997, ApJ, 481, 500

Cheng, J. X., Ding, M. D., \& Li, J. P. 2006, ApJ, 653, 733

de la Beaujardiere, J.-F., Kiplinger, A. L., \& Canfield, R. C. 1992, ApJ, 401, 761

de la Cruz Rodríguez, J., Löfdahl, M., Sütterlin, P., Hillberg, T., \& Rouppe van der Voort, L. 2015, A\&A, 573, A40

Deng, N., Tritschler, A., Jing, J., et al. 2013, ApJ, 769, 112

De Pontieu, B., McIntosh, S. W., Hansteen, V. H., \& Schrijver, C. J. 2009, ApJL, 701, L1 
Ding, M. D., \& Fang, C. 1996, SoPh, 166, 437

Falchi, A., Qiu, J., \& Cauzzi, G. 1997, A\&A, 328, 371

Fang, C., Henoux, J. C., \& Gan, W. Q. 1993, A\&A, 274, 917

Fisher, G. H., Canfield, R. C., \& McClymont, A. N. 1985, ApJ, 289, 414

Gan, W. Q., Rieger, E., \& Fang, C. 1993, ApJ, 416, 886

Graham, D. R., \& Cauzzi, G. 2015, ApJL, 807, L22

Heinzel, P., Karlický, M., Kotrč, P., \& Švestka, Z. 1994, SoPh, 152, 393

Huang, Z., Madjarska, M. S., Koleva, K., et al. 2014, A\&A, 566, A148

Hudson, H. S. 2011, SSRv, 158, 5

Hurford, G. J., Schmahl, E. J., Schwartz, R. A., et al. 2002, SoPh, 210, 61 Ichimoto, K., \& Kurokawa, H. 1984, SoPh, 93, 105

Ji, G. P., Kurokawa, H., Fang, C., \& Huang, Y. R. 1994, SoPh, 149, 195

Kerr, G. S., Simões, P. J. A., Qiu, J., \& Fletcher, L. 2015, arXiv:1508.03813v1

Li, H., You, J., Yu, X., \& Du, Q. 2007, SoPh, 241, 301

Lin, R. P., Dennis, B. R., Hurford, G. J., et al. 2002, SoPh, 210, 3

Meegan, C., Lichti, G., Bhat, P., et al. 2009, ApJ, 702, 791

Mein, P., Mein, N., Malherbe, J.-M., et al. 1997, SoPh, 172, 161

Milligan, R. O., \& Dennis, B. R. 2009, ApJ, 699, 968

Neupert, W. M. 1968, ApJL, 153, L59
Rubio da Costa, F., Kleint, L., Petrosian, V., Sainz Dalda, A., \& Liu, W. 2014, ApJ, 804, 56

Scharmer, G. B. 2006, A\&A, 447, 1111

Scharmer, G. B., Bjelksjo, K., Korhonen, T. K., Lindberg, B., \& Petterson, B. 2003a, Proc. SPIE, 4853, 341

Scharmer, G. B., Dettori, P. M., Lofdahl, M. G., \& Shand, M. 2003b, Proc. SPIE, 4853,370

Scharmer, G. B., Narayan, G., Hillberg, T., et al. 2008, ApJL, 689, L69

Schwartz, R. A., Csillaghy, A., Tolbert, A. K., et al. 2002, SoPh, 210, 165

Severny, A. B. 1968, in Mass Motions in Solar Flares and Related Phenomena, ed. Y. Oehman (Stockholm: Almqvist), 71

Švestka, Z. 1976, Solar Flares (Berlin: Springer)

Švestka, Z., Kopecký, M., \& Blaha, M. 1962, BAICz, 13, 37

Tang, F. 1983, SoPh, 83, 15

van Noort, M., Rouppe van der Voort, L., \& Löfdahl, M. G. 2005, SoPh, 228, 191

Vissers, G., \& Rouppe van der Voort, L. 2012, ApJ, 750, 22

Wülser, J. P., \& Marti, H. 1989, ApJ, 341, 1088

Yu, X.-X., \& Gan, W.-Q. 2006, ChA\&A, 30, 294 